\title{
Sonografiekurse nach den ÖGUM-Richtlinien
}

Abdomen

- 21.09.-22.09.2012, Klagenfurt: UltraschallGrundkurs „Abdomen“

- 14.11.-17.11.2012, Wien: Ultraschall-Grundkurs Abdomen, Leitung \& Auskunft: Prof. Dr. N. Gritzmann (Sono Seminare), Wien, Tel. u. Fax: 01/774747117, E-Mail: norbert.gritzmann@gmail.com; www.sonoseminare.com

19.09.-21.09.2012, Salzburg: Fortgeschrittenenkurs Abdomen

- 21.11.-23.11.2012, Salzburg: Grundkurs Abdomen, Leitung: OA DR. Hollerweger; kontakt@bhb-sonokurse.at

- 11.10.-13.10.2012, Hohenems: Abdomensonografie, Leitung \& Auskunft: OA Dr. Gehmacher; otto.gehmacher@lkhh.at

- 10.10.-12.10.2012, Linz: Grundkurs Abdomen

- 30.01.-01.02.2013, Linz: Aufbaukurs Abdomen

- 13.03.-15.03.2013, Linz: Grundkurs Abdomen, Leitung: OA Dr. Günter Schneider; Anmeldung \& Auskunft: Frau Kornelia Söllinger, Tel..: 0732/7676-4441, kornelia.soellinger@elisabethinen.or.at

Bewegungsapparat / Nervensystem

- 19.10.-20.10.2012, Innsbruck: Workshop: Sonografie des peripheren Nervensystems Leitung: Prof. Dr. H. Gruber; Auskunft: Karoline Volderauer; karoline.volderauer@i-med.ac.a

- 20.09.-22.09.2012, Wien: Interdisciplinary Musculoskeletal Ultrasound Course Vienna
Leitung \& Auskunft: Priv. Doz. Dr. SchuellerWeidekamm; claudia.schueller-weidekamm@ meduniwien.ac.at

Echokardiografie

- 04.10.-06.10.2012, Hernstein: Aufbaukurs perioperative Echokardiografie, Leitung \& Auskunft: Univ. Prof. Dr. Koinig; herbert.koinig@meduniwien.ac.at

Kopf / Hals / Gefäße

- 16.11.-17.11.2012, Wien: Farbdopplersonografiekurs „Halsgefäße und periphere GefäBe“, Leitung \& Auskunft: Univ.-Prof. Dr. N. Gritzmann; norbert.gritzmann@gmail.com

Pädiatrie

- monatlich 2012, Stolzalpe: Stütz- und Bewegungsapparat: Schwerpunkt Sonografie Säuglingshüfte, Leitung \& Auskunft: Prof. Dr. Graf; christine.puff@lkh-stolzalpe.at

- 15.11.-18.11.2012, Leoben: Pädiatrische Uttraschalldiagnostik (Abdomen, Small Parts) Leitung \& Auskunft: Dr. Schweintzger; gerolf. schweintzger@lkh-leoben.at

- 08.11.-10.11.2012, Linz: Echokardiografie Aufbaukurs, Leitung \& Auskunft: Dr. Tulzer; gerald.tulzer@gespag.at

12.10.-13.10.2012, Wien: Neonatale Ultraschalldiagnostik

09.11.-10.11.2012, Wien: Hüftsonografiekurs, Leitung \& Auskunft: Dr. Zoder; gerlinde. zoder@wienkav.at 